\title{
Kondo effect in complex mesoscopic structures
}

\author{
O. Entin-Wohlman ${ }^{a, b, c}$, A. Aharony ${ }^{a, b}$, and Y. Meir ${ }^{a, d}$ \\ ${ }^{a}$ Department of Physics, Ben Gurion University, Beer Sheva 84105, Israel \\ ${ }^{b}$ School of Physics and Astronomy, Raymond and Beverly Sackler Faculty of Exact Sciences, \\ Tel Aviv University, Tel Aviv 69978, Israel \\ ${ }^{c}$ Albert Einstein Minerva Center for Theoretical Physics, Weizmann Institute of Science, Rehovot 76100, Israel \\ The Ilse Katz Center for Meso- and Nano-Scale Science and Technology, Ben Gurion University, Beer Sheva 84105, Israel
}

(October 31, 2018)

\begin{abstract}
We study the Kondo effect of a quantum dot placed in a complex mesoscopic structure. Assuming that electronic interactions are taking place solely on the dot, and focusing on the infinite Hubbard interaction limit, we use a decoupling scheme to obtain an explicit analytic approximate expression for the dot Green function, which fulfills certain Fermi-liquid relations at zero temperature. The details of the complex structure enter into this expression only via the self-energy for the noninteracting case. The effectiveness of the expression is demonstrated for the single impurity Anderson model and for the T-shaped network.
\end{abstract}

\section{INTRODUCTION AND OUTLINE}

The single-impurity Anderson model (SIAM) has been a paradigm of a strongly correlated electron system. ${ }^{1}$ This seemingly simple model gives rise to dynamical screening of the local spin by the electrons in the Fermi sea, leading to a crossover from a weak coupling system at high temperatures $(T)$ to a strongly coupled one at low $T$, with the relevant temperature scale given by the Kondo temperature, $T_{K}$. The recent observation of the Kondo effect in quantum dots (QD's), whose parameters can be tuned continuously, and which allow probing of various properties, ${ }^{2}$ has yielded strong theoretical efforts in this direction. ${ }^{3}$ Recent experiments on a QD embedded on one branch of the Aharonov-Bohm interferometer ${ }^{4}$ created additional interest in Kondo effects in complex networks.

While the high- $T$ behavior of the SIAM can be adequately described by perturbation theory or poor-man scaling, ${ }^{5}$ and the low- $T$ behavior is described by Fermi liquid theory, ${ }^{6}$ there is no simple theory that describes the model's dynamical properties correctly in the whole temperature range, including both the high- and the low$T$ limits. This crossover has been described by the computationally demanding numerical renormalization group (NRG) approach. ${ }^{7}$ Other methods to describe the crossover are the "conserving T-matrix approximation" 8 (which overestimates the unitarity sum-rule), or the more limited "non-crossing approximation", ${ }^{9}$ and to some extent, quantum Monte Carlo calculations. ${ }^{10}$ Some of these methods have also been applied to the $\mathrm{QD}$ in the Aharonov-Bohm interferometer. ${ }^{11}$ However, none of these methods has the flexibility to follow analytically the effects of various network parameters (e.g. a magnetic flux) on experimentally measurable quantities, in particular dynamical ones.

In this paper we discuss the SIAM for a QD which is embedded in a general complex network. Many of the interesting physical properties of the system can be expressed explicitly in terms of the single electron (retarded) Green function (GF) on the dot, $G_{d d \sigma}(\omega)$, for electrons with spin component $\sigma$ and energy $\omega$ (measured relative to the Fermi energy). Here,

$$
\begin{aligned}
G_{A B \sigma}(\omega) & \equiv \ll A_{\sigma} ; B_{\sigma}^{\dagger} \gg \\
& \equiv-i \int_{0}^{\infty} d t e^{i(\omega+i \eta) t}\left\langle\left[A_{\sigma}(t), B_{\sigma}^{\dagger}\right]_{+}\right\rangle .
\end{aligned}
$$

(For the dot GF, $A=B=d_{\sigma}$, where $d_{\sigma}$ destroys an electron with spin $\sigma$ on the dot). One example concerns the local density of states (LDOS) on the dot,

$$
\rho_{d \sigma}(\omega)=-\Im\left[G_{d d \sigma}(\omega)\right] / \pi
$$

Another example is the conductance $\mathcal{G}$ between two leads which are connected to sites on the network. For a general mesoscopic structure, this (linear response) conductance has the form ${ }^{12}$

$$
\mathcal{G}=\frac{2 e^{2}}{h} \int d \omega\left(-\frac{\partial f}{\partial \omega}\right) \tilde{\mathcal{G}}(\omega)
$$

where $f(\omega)$ is the Fermi distribution function (we set the Fermi energy at $\omega=0$ ),

$$
f(\omega)=\frac{1}{e^{\omega / k T}+1},
$$

and $\tilde{\mathcal{G}}(\omega)$ is often given in terms of the retarded $G_{d d \sigma}$, the advanced $G_{d d \sigma}^{*}$ and non-interacting parameters characterizing the network. For the simple SIAM (dot and leads), $\tilde{\mathcal{G}}(\omega)$ is proportional to $\rho_{d \sigma}(\omega) .{ }^{13,14}$

Here we use the equations of motion method to derive a simple analytic approximate expression for $G_{d d \sigma}$, for a $\mathrm{QD}$ on a general network. Our approximate formula exhibits the correct behavior both at high and at very low temperatures. For simplicity, we consider a QD with a 
single level (of energy $\epsilon_{d}$ ). Electron-electron interactions are assumed to exist only on the QD, and we take their energy $U$ to be infinite. In our scheme, one first solves for the GF on the dot in the absence of the electron-electron interactions, i.e. $U=0$. This (spin-independent) GF can be written in the form

$$
G_{d d}^{0}(\omega)=\frac{1}{\omega+i \eta-\epsilon_{d}-\Sigma_{0}(\omega)},
$$

with the energy dependent (complex) self-energy (SE)

$$
\Sigma_{0}(\omega) \equiv \delta \epsilon_{d}(\omega)-i \Delta(\omega)
$$

This is easily done exactly, for any finite network; it only involves the solution of a finite set of linear equations. Our approximate explicit expression for $G_{d d \sigma}(\omega)$ is then given in a single equation [see Eq. (53) below]. Somewhat surprisingly, this equation depends on the network parameters only via $\Sigma_{0}(\omega)$. Although approximate, this expression allows for detailed systematic investigations of the Kondo effect as function of the system parameters, on a variety of complex networks. Even if sometimes only qualitatively correct, such systematic studies help to investigate new physical phenomena on a broad variety of mesoscopic systems. Furthermore, the dynamical mean field theory ${ }^{15}$ (which was developed to address the physics of the periodic Anderson and Hubbard models) iterates the local GF of the SIAM, which is calculated at each stage in terms of the effective SE created by the rest of the lattice. Since our GF is easily calculated in terms of $\Sigma_{0}$, it is an ideal candidate for such calculations. We are not aware of alternative simple analytic expressions which obey the necessary requirements at both high and low $T$.

In Sec. II we follow Refs. 16-19, and derive the equation-of-motion (EOM) for the dot GF. Extending these references, this EOM is derived here for a generalized case, in which the dot "sits" within an arbitrary complex network. The EOM for the dot GF involves higher-order GF's (consisting of more operators), whose EOM's introduce in turn more GF's. One then terminates this hierarchy by decoupling out averages of operators. The latter are then found using the fluctuationdissipation relationship, from the relevant GF's. In this manner, the treatment becomes self-consistent. The successful decoupling must keep as much of the electronic correlations as possible. ${ }^{17}$ For example, a widely used earlier approximation ${ }^{14}$ neglected some dot-lead correlations and therefore gave reasonable results only at $T>T_{K}$. For this reason, Gerland et al. ${ }^{20}$ had to combine the EOM method at high $T$ with the NRG at low $T$. After we correct these earlier calculations, and include all the necessary correlations, we obtain good results for all $T$. An earlier application of the EOM method solved the integral equations numerically, on the simplest interferometer geometry. ${ }^{21}$

The above decoupling scheme produces an integral equation for $G_{d d \sigma}(\omega)$, which generalizes that found in
Refs. 18 and 22. A new approximate analytical solution for this equation is found in Sec. III. The low temperature limit, the Fermi liquid conditions, and the Kondo behavior are discussed in Sec. IV. Unlike earlier papers (spanning over four decades), our solution gives good qualitative results for the whole parameter range: It has the correct high- $T$ behavior and fulfills several low- $T$ Fermi liquid relations.

For the simplest SIAM, the "network" is represented by a band with a density of states $N(\omega)$. In the broad band limit, the self-energy on the QD can be approximated by its value at the Fermi energy, $\Sigma_{0}(\omega) \approx-i \Delta_{b}=$ $-i \pi V^{2} N(0)$, and $\Delta_{b}$ represents the width of the impurity state in the absence of interactions ( $V$ represents the approximately energy-independent dot-band coupling). In this case, our equations reduce to those discussed in the earlier literature. We emphasize again that even the solution for this simple case is new and non-trivial, since earlier work either did not have a close analytical formula or missed some correlations, leading to wrong results at low $T$.

As stated, our result for $G_{d d \sigma}(\omega)$ depends on the details of the general network only via the non-interacting self-energy, $\Sigma_{0}(\omega)$. To demonstrate the power of our result, we present here a few simple examples. Section V contains explicit results for the simple case of the SIAM, when the QD is coupled to two one-dimensional leads. For this case, we easily calculate various features of the Kondo behavior, including the peak in $\rho_{d d \sigma}$ at the Fermi energy, the plateau in the conductance in the so-called unitary limit and the plateau in the "transmission phase" at $\pi / 2$.

A second example, of the so called "T" network, is solved in Sec. VI. In this case, one has interference between the wave function on the dot and that on the intersection point. Our simple approximate formula exhibits the Fano vanishing of the transmission and the associated so-called anti-Kondo effect, as seen experimentally. ${ }^{23}$ Here we also extend earlier theoretical work, which used complicated techniques. ${ }^{24,25}$ Finally, Sec. VII contains our summary.

\section{EQUATIONS OF MOTION}

\section{A. The Hamiltonian}

Our Hamiltonian,

$$
\mathcal{H}=\mathcal{H}_{\text {dot }}+\mathcal{H}_{\text {net }}+\mathcal{H}_{\text {dot-net }},
$$

contains the dot part

$$
\mathcal{H}_{d}=\epsilon_{d} \sum_{\sigma} n_{d \sigma}+U n_{d \uparrow} n_{d \downarrow},
$$

with a single energy level $\epsilon_{d}$ and an electron-electron interaction $U\left(n_{d \sigma}=d_{\sigma}^{\dagger} d_{\sigma}\right)$. All the other parts of $\mathcal{H}$ can be arbitrary, but non-interacting: 


$$
\mathcal{H}_{\mathrm{net}}=\sum_{n \sigma} \epsilon_{n} a_{n \sigma}^{\dagger} a_{n \sigma}-\sum_{n m \sigma} J_{n m} a_{n \sigma}^{\dagger} a_{m \sigma}
$$

describes the network without the dot, and

$$
\mathcal{H}_{\mathrm{dot}-\mathrm{net}}=-\sum_{n \sigma}\left(J_{d n} d_{\sigma}^{\dagger} a_{n \sigma}+\text { h.c. }\right)
$$

describes the coupling of the dot to the network. Here, $a_{n \sigma}^{\dagger}$ creates an electron with spin $\sigma$ and energy $\epsilon_{n}$ on the site $n$ of the network. The coefficients $J_{n m}$ and $J_{d n}$ represent tight-binding hopping matrix elements. In the absence of a magnetic flux, these coefficients can be chosen to be real. The flux then turns them complex, with phases which relate to the Aharonov-Bohm effect and with $J_{n m}=J_{m n}^{*} \cdot{ }^{11,21,26}$

The main part of this paper concerns the above general Hamiltonian, which assumes no specific details on the structure of the network. However, for some specific applications, it is convenient to attach a finite mesoscopic network to several semi-infinite leads, which connect to electron reservoirs. We thus divide $\mathcal{H}_{\text {net }}$ into two parts,

$$
\mathcal{H}_{\text {net }}=\mathcal{H}_{\text {meso }}+\mathcal{H}_{\text {leads }}
$$

where $\mathcal{H}_{\text {meso }}$ has the same form as $\mathcal{H}_{\text {net }}$, except that the sum is restricted to sites which belong to the finite mesoscopic part of the net (excluding the dot and the leads), and

$$
\begin{aligned}
\mathcal{H}_{\text {leads }} & =\sum_{L}\left[\mathcal{H}_{L}-\sum_{n_{L}, r}\left(J_{n_{L}, r} a_{n_{L} \sigma}^{\dagger} c_{r \sigma}^{(L)}+h . c .\right)\right], \\
\mathcal{H}_{L} & =-\sum_{r s} J_{r s} c_{r \sigma}^{(L) \dagger} c_{s \sigma}^{(L)}+\text { h.c.. }
\end{aligned}
$$

Here $n_{L}$ are the indices of the points on the mesoscopic structure which are connected to lead number $L$, while $c_{r \sigma}^{(L)}$ destroys an electron of spin $\sigma$ on the site $r$ of the lead.

Usually, the lead $L$ has a continuous spectrum, with eigenstates $|k\rangle$ and eigenenergies $\epsilon_{k}$, in a band of width $2 D$. Equation (12) can then be written as

$$
\begin{aligned}
\mathcal{H}_{\text {leads }} & =\sum_{L}\left[\mathcal{H}_{L}-\sum_{n_{L}, k}\left(V_{n_{L}}(k) a_{n_{L} \sigma}^{\dagger} c_{k \sigma}^{(L)}+h . c .\right)\right], \\
\mathcal{H}_{L} & =\sum_{k} \epsilon_{k} c_{k \sigma}^{(L) \dagger} c_{k \sigma}^{(L)},
\end{aligned}
$$

and also

$$
\begin{aligned}
\mathcal{H}_{\mathrm{dot}-\mathrm{net}} & =-\sum_{n \sigma}\left(J_{d n} d_{\sigma}^{\dagger} a_{n \sigma}\right. \\
& \left.-\sum_{L} \sum_{k \sigma} V_{d}(k) d_{\sigma}^{\dagger} c_{k \sigma}^{(L)}+\text { h.c. }\right),
\end{aligned}
$$

with obvious definitions. When the network consists only of the dot and the leads (i.e. $\mathcal{H}_{\text {meso }}=0$ ) then this has exactly the form of the standard SIAM model, discussed broadly in the literature. If all the leads have similar bands, then the electrons in the whole system also have a continuous spectrum in the range $-D<\omega<D$. All integrals over $\omega$ below will be thus over this energy band, and it is due to resonances in such integrals that we need to calculate the retarded GF at $\omega+i \eta$.

\section{B. Derivation of $\Sigma_{0}(\omega)$}

From now on we return to the general Hamiltonian, Eqs. (7-10). The EOM for the retarded GF on the QD reads

$$
\left(\omega+i \eta-\epsilon_{d}\right) G_{d d \sigma}=1-\sum_{n} J_{d n} G_{n d \sigma}+U \Gamma_{\sigma},
$$

where

$$
\Gamma_{\sigma}=\ll n_{d-\sigma} d_{\sigma} ; d_{\sigma}^{\dagger} \gg .
$$

From now on, we shall use the shorthand $\omega$ for $\omega+i \eta$, except where it matters. Specifically,

$$
\left(\omega-\epsilon_{n}\right) G_{n d \sigma}=-J_{d n}^{*} G_{d d \sigma}-\sum_{m} J_{n m} G_{m d \sigma} .
$$

We next define the matrix

$$
\mathcal{M}(\omega)_{n m} \equiv\left\langle n\left|\omega-\mathcal{H}_{\mathrm{net}}\right| m\right\rangle \equiv J_{n m}+\delta_{n m}\left(\omega-\epsilon_{n}\right),
$$

which represents the system without the dot. In the following, we shall need the inverse matrix,

$$
\mathcal{F}(\omega) \equiv[\mathcal{M}(\omega)]^{-1},
$$

which is the Green function for the system without the dot. With this definition one finds

$$
G_{m d \sigma}=u_{m}(\omega) G_{d d \sigma},
$$

where

$$
u_{m}(\omega) \equiv-\sum_{n} \mathcal{F}(\omega)_{m n} J_{n d} .
$$

We can now calculate the second term on the RHS of Eq. (15), and show that

$$
-\sum_{n} J_{d n} G_{n d \sigma}=\Sigma_{0} G_{d d \sigma}
$$

with

$$
\Sigma_{0}(\omega) \equiv-\sum_{m} J_{d m} u_{m}(\omega) \equiv \sum_{m n} J_{d m} \mathcal{F}(\omega)_{m n} J_{n d} .
$$

At $U=0$, Eq. (15) thus reduces to Eq. (5). Clearly, $\Sigma_{0}$ can be calculated for the non-interacting case.

All the other GF's can similarly be expressed in terms of $G_{d d \sigma}$. For example,

$$
G_{d m \sigma}=u_{m}^{*}\left(\omega^{*}\right) G_{d d \sigma},
$$


where $\omega^{*} \equiv \omega-i \eta$. Another way to represent $u_{m}^{*}\left(\omega^{*}\right)$ is to start from $u_{m}(\omega)$, and then take the complex conjugate only of the hopping coefficients $J_{m n}$ (namely reverse all their phases), without touching $\omega+i \eta$. Similarly,

$$
G_{\ell m \sigma}=\mathcal{F}(\omega)_{\ell m}+u_{\ell}(\omega) G_{d m \sigma} .
$$

Since $\mathcal{M}$ is an infinite matrix, its inversion may not be trivial. Formally, we can denote the eigenenergies and eigenstates of $\mathcal{H}_{\text {net }}$ by $\epsilon_{a}$ and $|a\rangle$, and then write

$$
\mathcal{F}(\omega)_{m n}=\sum_{a}\langle m \mid a\rangle \frac{1}{\omega+i \eta-\epsilon_{a}}\langle a \mid n\rangle .
$$

However, in the examples it is useful first to eliminate the parts which involve the sites on the leads. Examples for this procedure are presented in Appendix A.

\section{EOM's for higher-order GF's}

The EOM for $\Gamma_{\sigma}$ (with only on-site Hubbard interaction on the dot) does not involve GF's of more arguments:

$$
\begin{aligned}
\left(\omega-\epsilon_{d}-U\right) \Gamma_{\sigma} & =\left\langle n_{d-\sigma}\right\rangle+\sum_{n} J_{d n}^{*} \Gamma_{n \sigma}^{(3)} \\
& -\sum_{n} J_{d n}\left(\Gamma_{n \sigma}^{(1)}+\Gamma_{n \sigma}^{(2)}\right),
\end{aligned}
$$

with three new GF's,

$$
\begin{aligned}
& \Gamma_{n \sigma}^{(1)}=\ll n_{d-\sigma} a_{n \sigma} ; d_{\sigma}^{\dagger} \gg, \\
& \Gamma_{n \sigma}^{(2)}=\ll d_{-\sigma}^{\dagger} a_{n-\sigma} d_{\sigma} ; d_{\sigma}^{\dagger} \gg, \\
& \Gamma_{n \sigma}^{(3)}=\ll a_{n-\sigma}^{\dagger} d_{-\sigma} d_{\sigma} ; d_{\sigma}^{\dagger} \gg .
\end{aligned}
$$

The inhomogeneous term in Eq. (27), $\left\langle n_{d-\sigma}\right\rangle$, represents the average number of electrons with spin $\sigma$ on the dot. This number needs to be determined self-consistently, via the fluctuation-dissipation theorem:

$$
\left\langle n_{d \sigma}\right\rangle=\int d \omega f(\omega) \rho_{d \sigma}(\omega)
$$

We shall return to this condition below.

We now write the EOM's for the new GF's, $\Gamma_{n \sigma}^{(i)}$, and use several approximations for their solution. Firstly, it is easy to see from the EOM for $\Gamma_{n \sigma}^{(3)}$ that this GF is of order $1 / U$. Therefore, it contributes to $\Gamma_{\sigma}$ only at order $1 / U^{2}$, and can be ignored for $U \rightarrow \infty$ [we need $\Gamma_{\sigma}$ only to order $1 / U$, see Eq. (15)]. Secondly, we introduce a decoupling scheme for the new GF's that appear in the EOM's of the $\Gamma_{n \sigma}^{(i)}$ 's,

$$
\begin{aligned}
\ll a_{n \sigma_{1}}^{\dagger} a_{m \sigma_{2}} d_{\sigma_{3}} ; & d_{\sigma}^{\dagger} \gg \simeq\left\langle a_{n \sigma_{1}}^{\dagger} a_{m \sigma_{1}}\right\rangle \delta_{\sigma_{1} \sigma_{2}} \ll d_{\sigma_{3}} ; d_{\sigma}^{\dagger} \gg \\
& -\left\langle a_{n \sigma_{1}}^{\dagger} d_{\sigma_{1}}\right\rangle \delta_{\sigma_{1} \sigma_{3}} \ll a_{m \sigma_{2}} ; d_{\sigma}^{\dagger} \gg .
\end{aligned}
$$$$
\left(\omega-\epsilon_{d}-\Sigma_{0}\right) G_{d d \sigma}=1-\left\langle n_{d-\sigma}\right\rangle+G_{d d \sigma} \int\left(-\frac{d \omega^{\prime}}{2 \pi i}\right) \frac{f\left(\omega^{\prime}\right)}{\omega-\omega^{\prime}+i \eta}\left[\left(1+\Sigma_{0}^{\prime} G_{d d-\sigma}^{\prime}\right)\left(\Sigma_{0}^{\prime}-\Sigma_{0}\right)-\left(\omega^{\prime} \rightarrow \omega^{\prime *}\right)\right]
$$

We omit thermal averages of the form $\left\langle a_{n \sigma} d_{\sigma}\right\rangle$, which include two destruction (or creation) operators, and $\left\langle a_{n \sigma}^{\dagger} a_{m \sigma^{\prime}}\right\rangle$ with $\sigma \neq \sigma^{\prime}$, relevant only for states with a net magnetic moment. The latter assumption means that we restrict the discussion only to symmetric states, with $G_{d d \uparrow}=G_{d d \downarrow}$.

Using these approximations, the EOM for $\Gamma_{n \sigma}^{(1)}$ becomes

$$
\left(\omega-\epsilon_{n}\right) \Gamma_{n \sigma}^{(1)}=-J_{d n}^{*} \Gamma_{\sigma}-\sum_{m} J_{n m} \Gamma_{m \sigma}^{(1)} .
$$

The derivation of this equation also required an additional term, $-G_{n d \sigma}\left[\Sigma_{m} J_{d m}\left\langle d_{-\sigma}^{\dagger} a_{m-\sigma}\right\rangle-\right.$ c.c. $]$. However, this term vanishes. Here and below, we calculate equilibrium thermal averages by using the fluctuationdissipation theorem,

$$
\left\langle A^{\dagger} B\right\rangle=\int\left(-\frac{d \omega}{2 \pi i}\right) f(\omega)\left(G_{B A}-G_{A B}^{*}\right)
$$

Equation (31) is practically the same as Eq. (17), which was used to derive $\Sigma_{0}$ in terms of the $J_{n m}$ 's. Applying the same algebra to Eq. (31) then yields the ana$\log$ to Eq. (22),

$$
-\sum_{n} J_{d n} \Gamma_{n \sigma}^{(1)}=\Sigma_{0} \Gamma_{\sigma}
$$

Using the same approximations, the EOM of $\Gamma_{n \sigma}^{(2)}$ is

$$
\begin{aligned}
& \left(\omega-\epsilon_{n}\right) \Gamma_{n \sigma}^{(2)}=-J_{d n}^{*} \Gamma_{\sigma}-\sum_{m} J_{n m} \Gamma_{m \sigma}^{(2)} \\
& \quad+\left\langle X_{n}\right\rangle G_{d d \sigma}+\left\langle d_{-\sigma}^{\dagger} a_{n-\sigma}\right\rangle\left(1+\Sigma_{0} G_{d d \sigma}\right)
\end{aligned}
$$

in which

$$
\left\langle X_{n}\right\rangle=\sum_{m} J_{d m}^{*}\left\langle a_{m-\sigma}^{\dagger} a_{n-\sigma}\right\rangle
$$

Although the first two terms on the RHS of Eq. (34) are similar to those in Eq. (31), we now need to calculate the other two terms. Since these terms require thermal averages, which we express using the fluctuation-dissipation Eq. (32), they end up with integrals which involve $G_{d d-\sigma}$, leading finally to our integral equation for the dot GF. The details of these calculations are presented in Appendix B.

Inserting the results for the necessary thermal averages into the RHS of Eq. (34), the results are used in Eq. (B5) for the combination $-\sum_{n} J_{d n} \Gamma_{n \sigma}^{(2)}$ which is needed in the RHS of Eq. (27). Adding also the corresponding combination for $\Gamma^{(1)}$ [Eq. (33)], yields our final result for $\Gamma_{\sigma}$. It remains to insert it back into the EOM (15) for the dot Green function $G_{d d \sigma}$. This yields an integral equation for this function, 


$$
+\left(1+\Sigma_{0} G_{d d \sigma}\right) \int\left(-\frac{d \omega^{\prime}}{2 \pi i}\right) \frac{f\left(\omega^{\prime}\right)}{\omega-\omega^{\prime}+i \eta}\left[G_{d d-\sigma}^{\prime}\left(\Sigma_{0}-\Sigma_{0}^{\prime}\right)-\left(\omega^{\prime} \rightarrow \omega^{\prime *}\right)\right], \quad \eta \rightarrow 0^{+} .
$$

Here and below, $G_{d d \sigma}$ and $\Sigma_{0}$ are understood to be functions of $\omega \rightarrow \omega+i \eta$, and the primes denote a dependence on $\omega^{\prime} \rightarrow \omega^{\prime}+i \eta$, with $\omega^{\prime *} \rightarrow \omega^{\prime}-i \eta$. Two comments are in place here. First, for the simplest SIAM, when $\Sigma_{0}(\omega) \approx-i \Delta_{b}$ is independent of $\omega$, we have $\Sigma_{0}\left(\omega^{*}\right) \approx i \Delta_{b}$, so that only the parts with $\omega^{\prime *}$ survive. Furthermore, in this case one can factorize $\Delta_{b}$ out of the integrals. This reproduces the integral equation of Lacroix, ${ }^{18}$

$$
\begin{aligned}
\left(\omega-\epsilon_{d}+i \Delta_{b}\right) G_{d d \sigma} & =1-\left\langle n_{d-\sigma}\right\rangle-2 i \Delta_{b}\left[G_{d d \sigma} \int\left(-\frac{d \omega^{\prime}}{2 \pi i}\right) \frac{f\left(\omega^{\prime}\right)}{\omega-\omega^{\prime}+i \eta}\left(1+i \Delta_{b}\left[G_{d d-\sigma}^{\prime}\right]^{*}\right)\right. \\
& \left.-\left(1-i \Delta_{b} G_{d d \sigma}\right) \int\left(-\frac{d \omega^{\prime}}{2 \pi i}\right) \frac{f\left(\omega^{\prime}\right)}{\omega-\omega^{\prime}+i \eta}\left[G_{d d-\sigma}^{\prime}\right]^{*}\right] .
\end{aligned}
$$

However, even for this simple case there has not been an analytic solution that covers the whole parameter range. Second, discarding ${ }^{14}$ correlations between the dot and other sites on the net, e.g. $\left\langle d_{\sigma}^{\dagger} a_{n \sigma}\right\rangle$, amounts to neglecting the terms containing $G_{d d}^{\prime}$ in Eq. (36). This ends up with a breakdown of the Fermi liquid conditions at $T=0$, and with a bad approximation for $T<T_{K}$.

\section{APPROXIMATE SOLUTION OF THE INTEGRAL EQUATION}

We now restrict the discussion to "non-magnetic" states, and replace $G_{d d \uparrow}=G_{d d \downarrow} \equiv G_{d d}$. Equation (36) can then be written as

$$
\left(\omega-\epsilon_{d}-\Sigma_{0}\right) G_{d d}=\delta n+\mathcal{A} G_{d d}-\mathcal{B}\left(1-i \Delta G_{d d}\right)
$$

where $\delta n=1-\left\langle n_{d \uparrow}\right\rangle=1-\left\langle n_{d \downarrow}\right\rangle \equiv 1-\left\langle n_{d}\right\rangle / 2$ and $\mathcal{A}$ and $\mathcal{B}$ are functions of $\omega$, given by

$$
\begin{aligned}
& \mathcal{A}(\omega)=\int\left(-\frac{d \omega^{\prime}}{2 \pi i}\right) \frac{f\left(\omega^{\prime}\right)}{\omega-\omega^{\prime}+i \eta} \\
& \times\left[\Sigma_{0}^{\prime}\left(1+G_{d d}^{\prime}\left(\Sigma_{0}^{\prime}-\Sigma_{0}\right)\right)-\left(\omega^{\prime}+i \eta \rightarrow \omega^{\prime}-i \eta\right)\right] \\
& \mathcal{B}(\omega)=\int\left(-\frac{d \omega^{\prime}}{2 \pi i}\right) \frac{f\left(\omega^{\prime}\right)}{\omega-\omega^{\prime}+i \eta} \\
& \times\left[G_{d d}^{\prime}\left(\Sigma_{0}^{\prime}-\Sigma_{0}\right)-\left(\omega^{\prime}+i \eta \rightarrow \omega^{\prime}-i \eta\right)\right]
\end{aligned}
$$

For reasons that will be explained later, it is now convenient to replace $f\left(\omega^{\prime}\right)$ inside the integrals by $\left[f\left(\omega^{\prime}\right)-\right.$ $1 / 2]+1 / 2$. The part related to the constant $1 / 2$ can then be calculated using the Kramers-Kronig relations,

$$
\int\left(-\frac{d \omega^{\prime}}{2 \pi i}\right) \frac{1}{\omega-\omega^{\prime}+i \eta}\left(F\left(\omega^{\prime}\right)-F\left(\omega^{\prime}\right)^{*}\right) \equiv F(\omega),
$$

and thus we find

$$
\begin{aligned}
& \mathcal{A}(\omega)=\frac{1}{2} \Sigma_{0}(\omega)+\int\left(-\frac{d \omega^{\prime}}{2 \pi i}\right) \frac{f\left(\omega^{\prime}\right)-1 / 2}{\omega-\omega^{\prime}+i \eta} \\
& \times\left[\Sigma_{0}^{\prime}\left(1+G_{d d}^{\prime}\left(\Sigma_{0}^{\prime}-\Sigma_{0}\right)\right)-\left(\omega^{\prime}+i \eta \rightarrow \omega^{\prime}-i \eta\right)\right], \\
& \mathcal{B}(\omega)=\int\left(-\frac{d \omega^{\prime}}{2 \pi i}\right) \frac{f\left(\omega^{\prime}\right)-1 / 2}{\omega-\omega^{\prime}+i \eta}
\end{aligned}
$$

$$
\times\left[G_{d d}^{\prime}\left(\Sigma_{0}^{\prime}-\Sigma_{0}\right)-\left(\omega^{\prime}+i \eta \rightarrow \omega^{\prime}-i \eta\right)\right] .
$$

We next follow Lacroix, ${ }^{18}$ and assume that the integrals are dominated by the region $\omega^{\prime} \simeq \omega$, namely that

$$
\int d \omega^{\prime} F\left(\omega, \omega^{\prime}\right) \frac{f\left(\omega^{\prime}\right)-1 / 2}{\omega-\omega^{\prime}+i \eta} \simeq F(\omega, \omega) \mathrm{X}(\omega),
$$

and thus

$$
\mathcal{B}(\omega) \approx \Delta(\omega) G_{d d}^{*}(\omega) \mathrm{X}(\omega)
$$

where $\Delta(\omega)=-\Im \Sigma_{0}(\omega)$ and 22

$$
\begin{aligned}
\mathrm{X}(\omega) & =\int_{-D}^{D} \frac{d \omega^{\prime}}{\pi} \frac{f\left(\omega^{\prime}\right)-1 / 2}{\omega-\omega^{\prime}+i \eta} \\
& =\frac{1}{\pi}\left[\frac{1}{2} \ln \frac{\beta^{2}\left(D^{2}-\omega^{2}\right)}{(2 \pi)^{2}}-\Psi\left(\frac{1}{2}+\frac{\beta \omega}{2 \pi i}\right)\right],
\end{aligned}
$$

( $\Psi$ is the Digamma function). A similar procedure yields

$$
\mathcal{A}(\omega) \approx \Delta(\omega)\left[-\frac{i}{2}+X(\omega)\left(1+i \Delta(\omega) G^{*}(\omega)\right)\right]
$$

The reason for using the above transformation is that when one analyzes the equivalent equations for finite $U$, then one requires particle hole symmetry, namely the relation

$$
\widetilde{G}_{d d}(\omega)=-G_{d d}^{*}(-\omega),
$$

where the 'tilde' denotes particle-hole transformed quantities. In that case, this relation is equivalent to the relations

$$
\begin{aligned}
& \widetilde{\mathcal{B}}(\omega)=-\mathcal{B}^{*}(-\omega), \\
& \widetilde{\mathcal{A}}(\omega)=\mathcal{A}^{*}(-\omega)-\Sigma_{0}^{*}(-\omega) .
\end{aligned}
$$

These relations should hold also for any approximate solution. ${ }^{16}$ It is easy to check that these relations are obeyed by our approximate expressions (43) and (45), but would not hold if we made the approximation (42) on the original equations, before shifting $f\left(\omega^{\prime}\right)$ by $1 / 2 .{ }^{16,18}$ 
Taking $\delta n$ as a parameter, and defining the scaled variable

$$
z(\omega) \equiv \frac{\omega-\epsilon_{d}-\delta \epsilon_{d}(\omega)}{2 \Delta(\omega)}
$$

the solution of Eq. (38) can be written as

$$
G_{d d}(\omega)=g(\omega)\left(\delta n+i \mathrm{Q}(\omega) / \mathrm{X}^{*}(\omega)\right)
$$

with

$$
\begin{aligned}
& g=\frac{1}{\omega-\epsilon_{d}-\delta \epsilon_{d}+i 3 \Delta / 2} \equiv \frac{1}{2 \Delta(z+3 i / 4)}, \\
& \mathrm{Q}=\mathrm{S}-\left[\mathrm{S}^{2}+|\mathrm{X}|^{2}\left(\frac{3}{2} \delta n-\delta n^{2}\right)\right]^{1 / 2}, \\
& \mathrm{~S}=z^{2}+\frac{9}{16}-z \Re X+\left(\delta n-\frac{3}{4}\right) \Im X,
\end{aligned}
$$

and we have omitted the explicit dependence on $\omega$ for brevity. For some purposes it is more convenient to write Eq. (49) as

$$
G_{d d}(\omega)=\frac{\frac{3}{2}}{1-\mathcal{C}(\omega)} \frac{1}{\omega-\left(\epsilon_{d}+\delta E_{d}(\omega)\right)+i \frac{3}{2} \Delta(\omega)},
$$

where the real functions $\mathcal{C}(\omega)$ and $\delta E_{d}(\omega)$, which result from the strong interactions on the dot, depend on $T$ and on $\Sigma_{0}(\omega)$ and are given by

$$
\begin{aligned}
& \delta E_{d}(\omega)=\delta \epsilon_{d}-2 \Delta(\Re \mathrm{X}) \mathcal{C} /(1-\mathcal{C}) \\
& \mathcal{C}(\omega)=\frac{1}{2 \Delta^{2} Q\left|g\left(i+\delta n \mathrm{X}^{*} / Q\right)\right|^{2}} \equiv \frac{2\left(z^{2}+9 / 16\right)}{Q\left|i+\delta n \mathrm{X}^{*} / Q\right|^{2}}
\end{aligned}
$$

Equation (53) forms our general result. We emphasize again that all we need to know is the non-interacting self-energy, $\Sigma_{0}(\omega)$.

As seen explicitly from the above equations, our analytic expression for $G_{d d}$ depends on the parameter $\delta n=$ $1-\left\langle n_{d}\right\rangle / 2$, which needs to be determined self-consistently, via Eq. (29). We discuss this condition in a specific example below.

\section{THE FERMI LIQUID RELATIONS AND THE KONDO TEMPERATURE}

At $T=0$, one easily sees that

$$
\Re \mathrm{X}(\omega) \approx \ln |D / \omega| / \pi
$$

diverging at the Fermi energy $\omega \rightarrow 0$. At $T=\omega=0$ we thus have $\mathcal{C} \rightarrow 0$ and $\mathcal{C} \Re X \rightarrow u(z)$, with

$$
u(z)=\frac{2\left(z^{2}+9 / 16\right)\left[z+\sqrt{z^{2}+\delta n(1.5-\delta n)}\right]}{\left[z+\sqrt{z^{2}+\delta n(1.5-\delta n)}\right]^{2}+\delta n^{2}},
$$

and $z=z(\omega=0)=-\left[\epsilon_{s}+\delta \epsilon_{d}(0)\right] /[2 \Delta(0)]$. Therefore,

$$
G_{d d}(0)=\frac{1}{\Delta(0)((4 / 3)[z-u(z)]+i)} .
$$

For a very deep level on the dot, $z \gg 1, u(z)$ approaches $z$, and $G_{d d}$ approaches $-i / \Delta(0)$, ending up with high plateaus in the LDOS and in the phase of the complex $G_{d d}(\omega)$ sticking to $\pi / 2$. All of these characteristics, which are hallmarks of the Kondo behavior, ${ }^{20}$ are observed in the examples below. As $\epsilon_{d}$ moves up from large negative values, Eq. (57) shows a gradual crossover away from this "unitary" limit, and $G_{d d}$ becomes mainly real and small as $z \rightarrow-\infty$.

Eq. (57) also ensures that the GF obeys the Fermiliquid relations ${ }^{27}$ at $T=0$ : (i) The imaginary part of the SE on the Fermi level is the same as in the absence of interactions, i.e. $-\Delta(0)$, confirming the unitarity limit; ${ }^{28,13}$ (ii) For $\epsilon_{d} \ll-\Delta$, the LDOS there [i.e., $\left.\rho_{d}(\omega=0) \equiv \rho_{d \sigma}(0)\right]$ approaches the finite value $1 /[\pi \Delta(0)]$. We emphasize that these results apply for any complex network; the only input is $\Sigma_{0}(\omega)$.

The Kondo effect concerns the behavior of the density of states near the Fermi energy at low temperature. In that regime, $G_{d d}$ is dominated by its imaginary part, and thus

$$
\rho_{d} \approx 1 /[\pi \Delta(\omega)(1-\mathcal{C})]
$$

For $\Re X(\omega)>z(\omega) \gg 1$, rne has $S \approx z(z-\Re X)<0$, $Q \approx 2 S$ and the leading $\omega$-dependence of $\mathcal{C}$ is

$$
\mathcal{C} \sim \frac{z}{z-\Re X}
$$

We thus end up with a logarithmic cusp in $\rho_{d}$ at $\omega=0$. The related narrow peak reaches one half of its peak value (equal to $1 /[\pi \Delta(0)]$ ) when $\mathcal{C} \approx-1$. One definition of the Kondo energy $T_{K}$ is to identify $T_{K}$ with the half-width of this peak. Solving $\mathcal{C}\left(\omega= \pm T_{K}\right)=-1$ and using Eq. (59) thus yields $\Re X=2 z$, i.e.

$$
T_{K}\left(\epsilon_{d}\right)=D \exp \left[-a \pi\left|\epsilon_{d}+\delta \epsilon_{d}(0)\right| / \Delta(0)\right]
$$

with $a=1$. This result, which agrees with that of Lacroix, ${ }^{18}$ is qualitatively similar but quantitatively different from the presumably exact $T_{K}$ as given by Haldane, ${ }^{29}$ which has $a=1 / 2$. However, $T_{K}$ only represents some crossover energy scale, and we expect the above solution to follow the qualitative variations of $T_{K}$ with the system parameters, which are contained in $\delta \epsilon_{d}$ and in $\Delta$. It should be emphasized that $T_{K}$ depends on both $\Delta$ and $\delta \epsilon_{d}$ (some authors ignore the part coming from the real part of the SE, $\left.\delta \epsilon_{d}\right)$. For example, for the Aharonov-Bohm interferometer $T_{K}$ oscillates with the flux. ${ }^{30}$

At the Fermi energy, $\omega=0$, and for fixed $\epsilon_{d}<0$, one expects $\rho_{d}(0)$ to decrease with increasing $T$. In this limit, $X(\omega=0)=\ln |A D / T| / \pi$, with $A=e^{-\Psi(1 / 2)} /(2 \pi) \approx$ 
1.13387. For $\left|\epsilon_{d}\right| \gg \Delta(0)$ we then find the asymptotic relation

$$
\begin{aligned}
& \rho_{d}(0) \approx \frac{1}{\pi \Delta(0)(1-\mathcal{C})} \sim \frac{\Re X-z}{\pi \Delta(0) \Re X} \\
& =\frac{1}{\pi \Delta(0)}\left(1+\frac{\pi\left(\epsilon_{d}+\delta \epsilon_{d}\right)}{2 \Delta(0) \ln (A D / T)}\right) .
\end{aligned}
$$

This density of states decreases to one half of its maximum $T=0$ value at a temperature $A T_{K}$, with $T_{K}$ given in Eq. (60). Although the result (61) is qualitatively correct, and is also consistent with our $T_{K}$, its explicit low-temperature dependence disagrees with exact expectations: we obtain a logarithmic behavior, whereas Fermi liquid theory predicts a $T^{2}$ dependence at low $T{ }^{6,7}$ This is an artifact of our approximation. However, at high $T$ we do recover the usual logarithmic variation.

In the opposite limit, of $|z| \gg \Re X \sim 1$, one finds that $|Q / X| \ll 1$, and Eq. (49) implies that $G_{d d}(\omega)$ approaches $\delta n g(\omega)$. The appearance of $\delta n$ in the numerator, in place of 1 , results from the fact that some weight of the spectral function is pushed to infinite $U .{ }^{14}$ Another interesting point concerns the factor $3 / 2$ in Eqs. (50) and (53). This factor may be explained heuristically ${ }^{31}$ by the fact that while both spin directions are accessible for tunnelling into the dot, only a single one can tunnel out of it.

\section{EXAMPLE 1: THE SIMPLE SIAM}

In the following two examples, we assume simple semiinfinite one-dimensional leads, with identical nearestneighbor hopping matrix elements, $-J$, and with a lattice constant $a$. The eigenenergies of each $\mathcal{H}_{L}$ are therefore $\epsilon_{k}=-2 J \cos k a$, with eigenfunctions $\langle n \mid k\rangle=$ $\sin n k a \sqrt{2 / \Omega}(\Omega \rightarrow \infty$ is the length of the lead $)$, and $D=2 J$. We also assume that the leads $L$ and $R$ are attached to the mesoscopic network only at one site, with hopping matrix elements $J_{\ell}$ and $J_{r}$. For the simple SIAM, when the two leads are directly connected to the dot, we show in Appendix A that the leads generate a self-energy

$$
\Sigma_{0}(\omega)=-e^{i|q| a}\left(\left|J_{\ell}\right|^{2}+\left|J_{r}\right|^{2}\right) / J \equiv-e^{i|q| a} \Delta_{b}
$$

on the dot, with $q$ determined by $\omega=-2 J \cos q a$ and with

$$
\Delta_{b} \equiv \Gamma_{\ell}+\Gamma_{r} .
$$

Here we have used the notation

$$
\Gamma_{\ell, r} \equiv\left|J_{\ell, r}\right|^{2} / J=\pi \mathcal{N}(0)\left|J_{\ell, r}\right|^{2},
$$

with $\mathcal{N}(\omega)$ being the density of the band states. The width of the non-interacting resonance at $\epsilon_{d}$ is thus equal to $\Delta_{b} \sin q a$. In the figures presented below we use the symmetric case, $\Gamma_{\ell}=\Gamma_{r}=\Delta_{b} / 2$. Substituting
$\Sigma_{0}(\omega)=\delta \epsilon_{d}(\omega)-i \Delta(\omega)$ into Eqs. (49)-(52) then yields $G_{d d}$.

We start by discussing the self-consistency condition for $\left\langle n_{d}\right\rangle$. Figure 1 shows an example of our self-consistent solutions for $\left\langle n_{d}\right\rangle$, at $T=0$ : we start from an initial guess for $\delta n$, then calculate $\left\langle n_{d}\right\rangle$ from Eq. (29), and iterate; the procedure converges after a few iterations. Also shown is $\tilde{n}$, which represents the total change in the electron occupation in the system due to the dot, as determined from the Friedel phase, defined via ${ }^{13,28,27}$

$$
\tan \left[\frac{\pi}{2}(1-\tilde{n})\right] \equiv \Re\left[G_{d d}(0)\right] / \Im\left[G_{d d}(0)\right] .
$$

For $\epsilon_{d} \ll-\Delta(0)=-\Delta_{b}, \tilde{n}$ approaches 1 , while $\left\langle n_{d}\right\rangle$ remains slightly smaller. This small difference could reflect an additional occupation of other sites in the leads. It could also result from the inaccuracy of our approximation for $G_{d d}(\omega)$, which becomes worse as $\omega$ moves away from the Fermi energy; the integral in Eq. (29) contains contributions from all $\omega$. However, this small difference has only a small effect on the other calculations presented below.

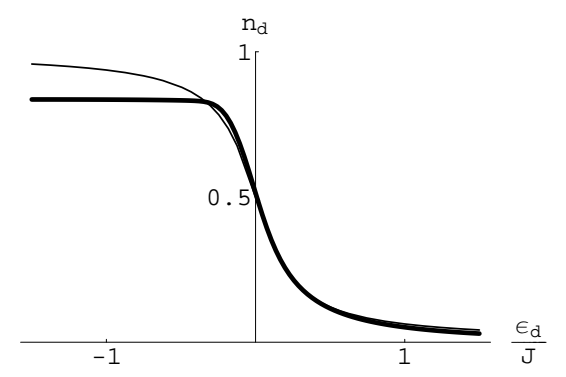

FIG. 1. The self-consistent average occupation on the dot, $\left\langle n_{d}\right\rangle$ (thick line), and the Friedel added occupation $\tilde{n}$ at $T=0$, as function of $\epsilon_{d}$, for the simplest SIAM with $\Delta_{b} / J=0.1$. Results remain almost unchanged up to $T \sim \Delta_{b}$.

Although it is easy to solve for $\left\langle n_{d}\right\rangle$ for each set of parameters and use the resulting $\delta n$ for other calculations, the qualitative results are only weakly affected if one uses an arbitrary smooth variation of $\left\langle n_{d}\right\rangle$ from 0 to 1 as $\epsilon_{d}$ varies from $+\infty$ to $-\infty$. In the calculations below, we used such a smooth variation for $\delta n$, with a width of order $\Delta_{b}$.

An alternative approximate expression for $\delta n$ follows from Eq. (49). It turns out that for most of the integration range in Eq. $(29), G_{d d}(\omega)$ is dominated by the first term there. Neglecting the second term, one has $1-\delta n=\left\langle n_{d \sigma}\right\rangle \approx \delta n n_{0}$, with $n_{0}=-\int d \omega \Im[g(\omega)] / \pi$ depending only on the non-interacting parameters. Thus, $\delta n \approx 1 /\left(1+n_{0}\right)$. For $\Delta_{b}$ between 0.1 and 0.01 and for $\epsilon_{d}<\Delta_{b}$, we find this estimate to be within $\sim 10 \%$ from the full self-consistent value.

Figure 2 presents the LDOS [Eq. (2)] versus $\omega$, for the simple SIAM, with the parameters as indicated. Plots at lower $T$ are indistinguishable from the one shown at 
$T=.01 J$. Our approximation reproduces the Kondo peak at low $T$, in addition to the much broader peak at $\epsilon_{d}$. Figure 3 shows $\rho_{d}$ at the Fermi energy $(\omega=0)$ versus $\epsilon_{d}$. Note the very slow (logarithmic) increase of $\rho_{d \sigma}$ with decreasing $T$, in agreement with Eq. (61).
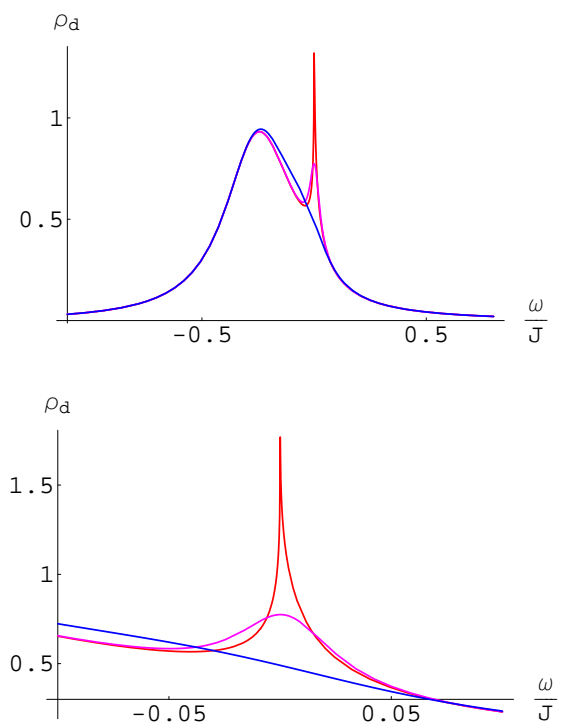

FIG. 2. The LDOS on the dot, for the simple SIAM, with $\epsilon_{d}=-.3, \Delta_{b}=.1$ and $T=0, .01, .05$ (all energies are in units of $J$ ). The Kondo peak values near $\omega=0$, shown with more detail in the lower panel, decrease with increasing $T$.

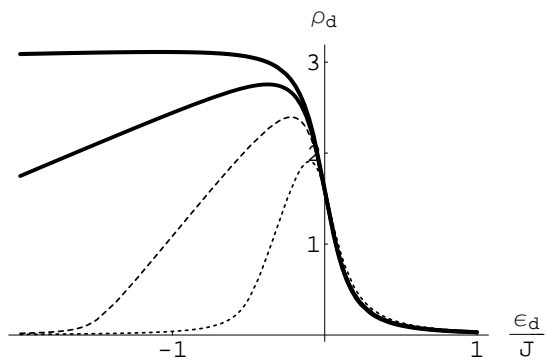

FIG. 3. The LDOS at the Fermi energy, $\rho_{d}(0)$, versus $\epsilon_{d}$, for $T=0,10^{-30}, 10^{-10}, 10^{-3}$ and $\Delta_{b}=.1 ; \rho_{d}(0)$ increases with decreasing $T$.

We now turn to the conductance, $\mathcal{G}$. For the simple SIAM, one has ${ }^{13,14}$

$$
\tilde{\mathcal{G}}(\omega)=-\frac{4 \Gamma_{\ell} \Gamma_{r}}{\Gamma_{\ell}+\Gamma_{r}} \Im G_{d d}(\omega)
$$

Equation (53) implies that

$$
\Im G_{d d}=-(1-\mathcal{C}) \Delta_{b}\left|G_{d d}\right|^{2} .
$$

For $T=0$, when $\mathcal{C} \rightarrow 0$, we also have $\tilde{\mathcal{G}}(\omega)=$ $4 \Gamma_{\ell} \Gamma_{r}\left|G_{d d}\right|^{2}$. At finite temperatures, these two expressions for the conductance exhibit the same qualitative behavior as $\rho_{d}$ (Fig. 3). The quantitative difference between the two expressions, which increases with $T$ and with large negative $\epsilon_{d}$, represents a breakdown of unitarity which may be an artifact of our approximation.

At low $T,(-\partial f / \partial \omega)$ is practically a delta function, and Eq. (3) yields $\mathcal{G} \approx\left(2 e^{2} / h\right) \tilde{\mathcal{G}}(0)$, exhibiting the qualitative behavior shown in Fig. 3. At higher $T$, the peak in $\mathcal{G}$ slightly below $\epsilon_{d}=0$ becomes lower and broader than that of $\rho_{d}(\omega=0)$. At fixed $\epsilon_{d}<0$, increasing $T$ results with a decreasing $\mathcal{G}$, but with an interesting superimposed peak at $T=\mathcal{O}\left(\left|\epsilon_{d}\right|\right.$ ) (when the peak at $\epsilon_{d}$ starts to contribute).

Some experiments ${ }^{4}$ place the QD on one branch of an open Aharonov-Bohm interferometer, and attempt to extract the transmission phase for scattering through the dot. The transmission phase is usually related to the phase of $G_{d d} .{ }^{20}$ Without getting into the question of what is really measured in the interferometer, ${ }^{32}$ it is still of interest to study the latter phase. Figure 4 thus shows the "transmission", represented by $\Delta_{b}^{2}\left|G_{d d}\right|^{2}$, and the "transmission phase", represented by [see Eq. (65)] $\alpha=\pi(1-\tilde{n} / 2)$ as function of $\epsilon_{d}$ for several temperatures. Interestingly, at high $T$ this phase simply grows smoothly from zero to $\pi$ through the resonance, similarly to the non-interacting case or to the Coulomb blockade case. However, as $T$ decreases, the peak in the "transmission" broadens towards negative $\epsilon_{d}$, eventually reaching a plateau for $T=0$ (see also Fig. 3 ). At the same time, the phase develops an intermediate plateau at $\pi / 2 .^{20}$ This plateau begins at an energy $\epsilon_{d}$ which is roughly given by $T \sim T_{K}\left(\epsilon_{d}\right)$, as defined in Eq. (60). Studying the energy where this phase grows from zero to $\pi / 2$ thus suggests another way to define the crossover temperature $T_{K}$.
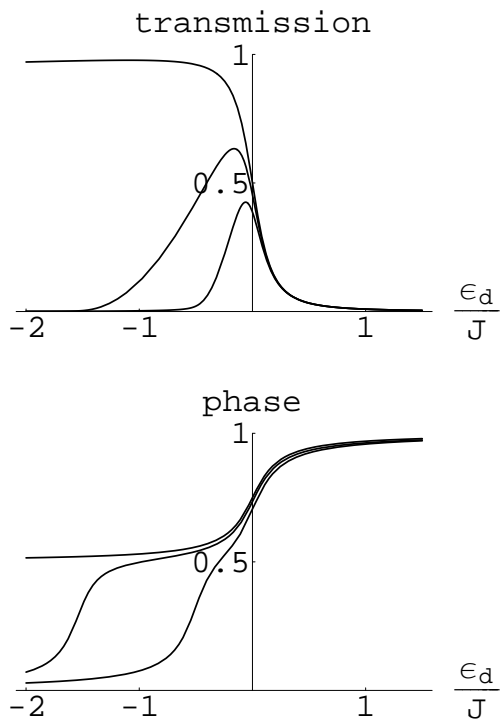

FIG. 4. "Transmission" and "transmission phase" (in units of $\pi$ ) for the simple SIAM, for $\Delta_{b}=0.1$ and for $T=0,10^{-10}, 10^{-3}$. 


\section{EXAMPLE 2: THE QD ON A "T" NETWORK}

Our equations also become very simple for the "T" network, when the dot sits on a side branch. Such a network has recently attracted both theoretical ${ }^{24,25}$ and experimental ${ }^{23}$ interest, as the simplest realization of the Fano-Kondo effect. The mesoscopic network now consists of a single site " 0 ". This site has one bond connected to the dot, with hopping $J_{x}$, and two bonds connected to two leads, with hopping $J_{\ell}$ and $J_{r}$. In this case, the matrix $F_{11}$ in Appendix A is of order $1 \times 1$, and Eqs. (A3) and (A8) yield $\mathcal{F}_{00}=1 /\left[\omega-\epsilon_{0}+e^{i|q| a}\left(\left|J_{\ell}\right|^{2}+\left|J_{r}\right|^{2}\right) / J\right]$. Using $\Delta_{b}$ from Eq. (63), we have

$$
\Sigma_{0}(\omega)=\frac{\left|J_{x}\right|^{2}}{\omega-\epsilon_{0}+e^{i|q| a} \Delta_{b}} \equiv \delta \epsilon_{d}(\omega)-i \Delta(\omega) .
$$

In this case, $\tilde{\mathcal{G}}$ of Eq. (3) is given by ${ }^{25}$

$$
\tilde{\mathcal{G}}(\omega)=-\frac{4 \Gamma_{\ell} \Gamma_{r}}{\Gamma_{\ell}+\Gamma_{r}} \Im G_{00}(\omega) .
$$

The calculation of $G_{00}$ follows directly from Eqs. and (25),

$$
G_{00}=\frac{1+\Sigma_{0} G_{d d}}{\omega-\epsilon_{0}+i \Delta_{b}}
$$

Therefore, $\Im G_{00}$ is a linear combination of $\Re G_{d d}$ and of $\Im G_{d d}$, with coefficients which depend only on the noninteracting parameters.

These equations reproduce those found e.g. in Refs. 24 and 25. However, at this point those authors use complicated numerical schemes to obtain $G_{d d}$, which do not allow for systematic studies of the dependence on the various parameters. In contrast, we can easily obtain the approximate $G_{d d}$ analytically, with the same ease as for the previous example. All we need to do is to substitute Eq. (68) into Eqs. (49)-(52).

For the non-interacting case, $G_{d d}$ is simply given by Eq. (5). Figure 5 then compares the results for the conductance (in units of $2 e^{2} / h$ ), with and without interactions. Without interactions (thin lines), for $\epsilon_{0}=0$ the upper panel in Fig. 5 shows a symmetric Fano vanishing of the conductance at $\epsilon_{d}=0$. As $\epsilon_{0}$ becomes more negative (lower panel there), the conductance assumes a typical non-symmetric Fano shape, with smaller conductances at large $\left|\epsilon_{d}\right|$. The interactions (thick lines) have a negligible effect at $\epsilon_{d} \gg 0$, when the dot is not occupied. However, at $\epsilon_{d}<0$ and $T=0$ (thickest lines) the interactions cause strong changes. For $\epsilon_{0}=0$ and large negative $\epsilon_{d}$, the occupation on the dot causes the so-called anti-Kondo effect, ${ }^{24}$ where the formation of the Kondo singlet causes destructive interference which yields zero conductance. For $\epsilon_{0}<0$, the Fano zero is shifted to lower $\epsilon_{d}$, the peak disappears and the asymptotic conductance increases. As $T$ increases (medium thick lines), the behavior at negative $\epsilon_{d}$ gradually returns to that of the non-interacting case, and the Fano zero disappears: the conductance is always non-zero. Again, all of these phenomena are qualitatively similar to those found in Refs. 23,24 and 25 .
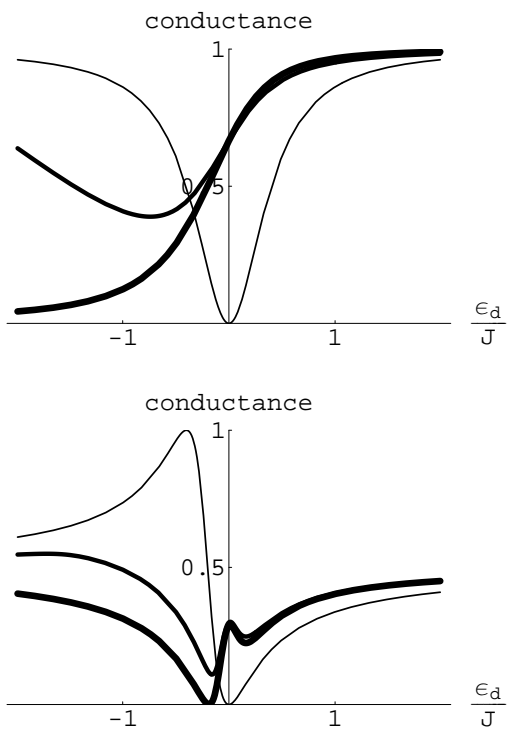

FIG. 5. Conductance of the "T" network, with $J_{x}=.2, \Delta_{b}=.1$ (all in units of $J$ ). Thin lines: without interactions. Thick lines: with infinite interactions at $T=0$ (intermediate thickness lines: $T=10^{-5}$ ). Upper panel: $\epsilon_{0}=0$. Lower panel: $\epsilon_{0}=-.1$.

A slightly more complex T-network is obtained by placing another non-interacting site, " 1 ", between the site 0 and the dot, with energy $\epsilon_{1}$ and with hopping elements $J_{x}$ to both site 0 and the dot. Our mesoscopic system now contains two sites, 0 and 1, and the methods of Appendix A yield

$$
\begin{gathered}
\Sigma_{0}=\frac{J_{x}^{2}\left(\omega-\epsilon_{0}+e^{i|q| a} \Delta_{b}\right)}{D}, \\
G_{00}=\frac{\omega-\epsilon_{1}}{D}+\frac{J_{x}^{4}}{D^{2}} G_{d d},
\end{gathered}
$$

with

$$
D=\left(\omega-\epsilon_{1}\right)\left(\omega-\epsilon_{0}+e^{i|q| a} \Delta_{b}\right)-J_{x}^{2}
$$

Figure 6 shows a few examples on how the interplay between $\epsilon_{0}$ and $\epsilon_{1}$ can change the dependence of the conductance on $\epsilon_{d}$ for the fully interacting case at $T=0$. For $\epsilon_{1}=\epsilon_{0}=0$, the graph looks exactly like that for the simple SIAM, discussed in Sec. V. Clearly, the presence of the intermediate point turned the destructive interference into a constructive one. Changing $\epsilon_{0}$ to positive (negative) values then simply shifts the whole curve to the left (right). Changing $\epsilon_{1}$ to non-zero values generates either a "Fano" zero in the conductance (when 
$\left.\epsilon_{0}<0\right)$, similar to the results in Fig. 5, or a "Fano" resonance (when $\epsilon_{0}>0$ ). At $\epsilon_{0}=0$ one observes a change from a zero to a resonance as $\epsilon_{1}$ changes from positive to negative values. All of these parameters can be easily changed using the various gate voltages, e.g in the setup of Ref. 23. Without going into much further discussion, it is clear that the intermediate point on the side branch is very effective in changing the interference pattern between Fano-Kondo resonances and anti-resonances. Data from such experiments can then be used to obtain information on $G_{d d}$.
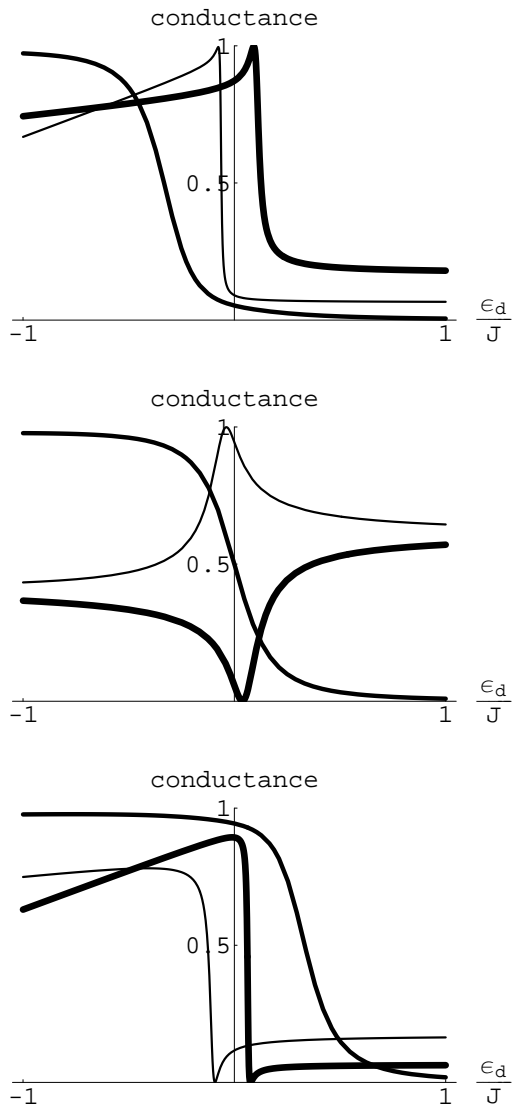

FIG. 6. Conductance of the "T" network, with an extra site "1" between the dot and the "intersection" site "0". Plots are for $U=\infty, T=0, J_{x}=.2, \Delta_{b}=.1$ (all in units of $J$ ). From top to bottom: $\epsilon_{0}=0.3,0,-0.3$. For each value of $\epsilon_{0}$, the figure contains graphs for $\epsilon_{1}=-0.5,0,0.5$, with increasing thickness.

\section{DISCUSSION}

We have derived an approximate analytic expression for a complex mesoscopic network, which contains a quantum dot with electron-electron interactions, and which may connect to several leads. Our formulae correct and generalize earlier expressions, and give a good qualitative interpolation between the Fermi liquid behavior at very low temperatures and the simpler high temperature one.

Although our formulae reproduce many features required by the Fermi liquid theory, they are still approximate, and should thus only be used for discussing the qualitative variation of various quantities on the parameters characterizing the network. However, the simplicity of our expressions allows for relatively easy comparisons with experiments and with more complicated numerical work.

We have demonstrated the use of our formulae for the two simple cases of a single quantum dot attached to two leads and of the "T" network, where the dot is connected to another site which couples to the two leads. Indeed, we have reproduced and extended all the expected phenomena for these two cases. In an upcoming publication ${ }^{30}$ we shall apply this scheme to the interesting case of the Aharonov-Bohm interferometer.

\section{ACKNOWLEDGEMENTS}

We acknowledge helpful discussions with A. Schiller, and support to YM from the Israel Science Foundation (ISF). This project was carried out in a center of excellence supported by the ISF (OE and AA). 


\section{APPENDIX A: CALCULATING $\mathcal{F}=\mathcal{M}^{-1}$}

We need to invert the matrix $\mathcal{M}=\omega+i \eta-\mathcal{H}_{\text {net }}$. We start by dividing our Hilbert space into two parts, called "1" and "2". Then, we write

$$
\mathcal{M}=\left(\begin{array}{ll}
M_{11} & M_{12} \\
M_{21} & M_{22}
\end{array}\right)
$$

and the inverse matrix $\mathcal{F}$ as

$$
\mathcal{F}=\left(\begin{array}{ll}
F_{11} & F_{12} \\
F_{21} & F_{22}
\end{array}\right)
$$

It is now easy to obtain the Dyson-like equation,

$$
F_{11}=\left(M_{11}-M_{12}\left[M_{22}\right]^{-1} M_{21}\right)^{-1} .
$$

In our calculations we only need $\mathcal{F}_{m n}$ for $m, n$ within the finite mesoscopic part of the network (possibly including the dot). Identifying this part with the subspace "1" above, we thus only need the matrix elements of the finite matrix $F_{11}$. To obtain these via Eq. (A3), we need the Green function for the leads, $\left[M_{22}\right]^{-1}$. For our Eq. (12), the matrix $M_{22}$ separates for the different leads, and we end up with

$$
F_{22}=\left[M_{22}-\sum_{L} \Sigma^{(L)}\right]^{-1}
$$

with the self-energy matrices

$$
\Sigma_{m n}^{(L)}=-\sum_{r s} J_{m r} g_{r s}^{(L)} J_{s m} .
$$

Here, the matrices $M_{22}$ and $\Sigma^{(L)}$ are all of order $N \times N$, where $N$ is the number of sites in the mesoscopic part of the network. Also,

$$
g_{r s}^{(L)}=\sum_{k} \frac{\langle r \mid k\rangle\langle k \mid s\rangle}{\omega-\epsilon_{k}}
$$

is the Green function for the disconnected lead $L$.

For the one-dimensional leads, we use the identity

$$
\frac{2}{\Omega} \sum_{k} \frac{1}{\omega+i \eta-\epsilon_{k}}=\frac{1}{2 i J \sin |q| a}
$$

with $\omega=-2 J \cos q a$, to obtain the self-energy due to the lead $L$, which is attached at the single point $n_{L}$,

$$
\Sigma^{(L)}(\omega)_{m n}=-\left|J_{\ell}\right|^{2} e^{i|q| a} \delta_{m, n_{L}} \delta_{m, n_{L}} / J .
$$

The same result also applies when the mesoscopic part is empty, and the leads are connected directly to the dot.

\section{APPENDIX B: DETAILS OF THE EOM FOR $\Gamma^{(2)}$}

Following the same logic that led to Eqs. (22) and (33), the formal solution to Eq. (34) gives

$$
\begin{aligned}
-\sum_{n} J_{d n} \Gamma_{n \sigma}^{(2)} & =\Sigma_{0} \Gamma_{\sigma}-\sum_{m n} J_{d m} \mathcal{F}_{m n}\left[\left\langle X_{n}\right\rangle G_{d d \sigma}\right. \\
& \left.+\left\langle d_{-\sigma}^{\dagger} a_{n-\sigma}\right\rangle\left(1+\Sigma_{0} G_{d d \sigma}\right)\right] .
\end{aligned}
$$

Calculating the thermal average $\left\langle d_{-\sigma}^{\dagger} a_{n-\sigma}\right\rangle$ via the fluctuation-dissipation Eq. (32), and using Eqs. (20) and (24), we end up with an integral which requires the sum

$$
S_{1}=\sum_{m n \ell} J_{d m} \mathcal{F}(\omega)_{m \ell} \mathcal{F}\left(\omega^{\prime}\right)_{\ell n} J_{n d} .
$$

For this and for similar sums, it is helpful to use the identity

$$
\mathcal{F}(\omega+i \eta) \mathcal{F}\left(\omega^{\prime} \pm i \eta^{\prime}\right)=\frac{\mathcal{F}\left(\omega^{\prime} \pm i \eta^{\prime}\right)-\mathcal{F}(\omega+i \eta)}{\omega-\omega^{\prime}+i \eta},
$$

which follows from Eq. (26), together with a careful use of $1 /(x+i \eta)=\mathcal{P}(1 / x)-i \pi \delta(x)$. Using Eq. (23), the sum in Eq. (B2) then becomes

$$
S_{1}=\frac{1}{\omega-\omega^{\prime}+i \eta}\left[\Sigma_{0}\left(\omega^{\prime}\right)-\Sigma_{0}(\omega)\right]
$$

Similar manipulations allow the calculation of the thermal averages $\left\langle X_{n}\right\rangle$. Finally, we end up with

$$
\begin{aligned}
-\sum_{n} J_{d n} \Gamma_{n \sigma}^{(2)} & =\Sigma_{0} \Gamma_{\sigma}+G_{d d \sigma} \int\left(-\frac{d \omega^{\prime}}{2 \pi i}\right) \frac{f\left(\omega^{\prime}\right)}{\omega-\omega^{\prime}+i \eta}\left[\left(1+\Sigma_{0}^{\prime} G_{d d-\sigma}^{\prime}\right)\left(\Sigma_{0}-\Sigma_{0}^{\prime}\right)-\left(\omega^{\prime} \rightarrow \omega^{\prime *}\right)\right] \\
& -\left(1+\Sigma_{0} G_{d d \sigma}\right) \int\left(-\frac{d \omega^{\prime}}{2 \pi i}\right) \frac{f\left(\omega^{\prime}\right)}{\omega-\omega^{\prime}+i \eta}\left[G_{d d-\sigma}^{\prime}\left(\Sigma_{0}-\Sigma_{0}^{\prime}\right)-\left(\omega^{\prime} \rightarrow \omega^{\prime *}\right)\right] .
\end{aligned}
$$

As explained after Eq. (36), $G_{d d \sigma}$ and $\Sigma_{0}$ are understood to be functions of $\omega \rightarrow \omega+i \eta$, and the primes denote a dependence on $\omega^{\prime} \rightarrow \omega^{\prime}+i \eta$, with $\omega^{\prime *} \rightarrow \omega^{\prime}-i \eta$.

\footnotetext{
${ }^{1}$ For a review, see e.g. P. Fulde, J. Keller and G. Zwicknagel, Solid State Phys. 41, 1 (1988).

${ }^{2}$ D. Goldhaber-Gordon, H. Shtrikman, D. Mahalu, D. Abusch-Magder, U. Meirav and M. A. Kastner, Nature 391, 156 (1998).
} 
${ }^{3}$ For a review, see e.g. N. S. Wingreen, Materials Science and Engineering B 84, 22 (2001).

${ }^{4}$ W. G. van der Wiel, S. De Franceschi, T. Fujisawa, J. M. Elzerman, S. Tarucha, and L. P. Kouwenhoven, Science 289, 2105 (2000); Y. Ji, M. Heiblum, D. Sprinzak, D. Mahalu and H. Shtrikman, Science 290, 779 (2000); Y. Ji, M. Heiblum and H. Shtrikman, Phys. Rev. Lett. 88, 076601 (2002).

${ }^{5}$ See, e.g., N. Sivan and N. S. Wingreen, Phys. Rev. B 54, 11622 (1996) and references therein.

${ }^{6}$ P. Noziéres, J . Low Temp. Phys. 17, 31 (1974); K. Yamada, Prog. Theor. Phys. 53, 970 (1975); 54, 316 (1975).

7 T. A. Costi and A. C. Hewson, J. Phys.: Cond. Matter 5, L361 (1993).

${ }^{8}$ S. Kirchner, J. Kroha, and P. Wölfle, Phys. Rev. B 70, 165102 (2004).

${ }^{9}$ N. E. Bickers, D. L. Cox and J. W. Wilkins, Phys. Rev. B 36, 2036 (1987); Q. Qin and H. Keiter, Z. Phys. B 65, 89 (1991).

10 J. E. Hirsch and R. M. Fye, Phys. Rev. Lett. 56, 2521 (1986); R. N. Silver, J. E. Gubernatis, D. S. Sivia and M. Jarrell, Phys. Rev. Lett. 65, 496 (1990).

${ }^{11}$ W. Hofstetter, J. König, and H. Schoeller, Phys. Rev. Lett. 87, 156803 (2001); J. König and Y. Gefen, Phys. Rev. Lett. 86, 3855 (2001); Phys. Rev. B 65, 045316 (2002); C. H. Lewenkopf and W. A. Weidenmüller, cond-mat/0401523.

12 O. Entin-Wohlman, Y. Imry, and A. Aharony, Phys. Rev. B 70, 075301 (2004).

${ }^{13}$ T. K. Ng and P. A. Lee, Phys. Rev. Lett. 61, 1768 (1988).

${ }^{14}$ Y. Meir, N. S. Wingreen, and P. A. Lee, Phys. Rev. Lett. 66, 3048 (1991).

${ }^{15}$ For a recent review, see e.g. A. George, cond-mat/0403123,

${ }^{16}$ L. Dworin, Phys. Rev. 164, 818 (1967). This paper shows that all self-consistent methods, to any order of truncation, have difficulties near the particle-hole symmetry point. Such difficulties should not show up in the limit $U \rightarrow \infty$ discussed here.

17 J. A. Appelbaum and D. R. Penn, Phys. Rev. B 3, 942 (1971).

18 C. Lacroix, J. Phys. F 11, 2389 (1981).

${ }^{19}$ H-G. Luo, Z-J. Ying, and S-J. Wang, Phys. Rev. B 59, 9710 (1999).

${ }^{20}$ U. Gerland, J. von Delft, T. A. Costi and Y. Oreg, Phys. Rev. Lett. 84, 3710 (2000).

${ }^{21}$ B. R. Bulka and P. Stefański, Phys. Rev. Lett. 86, 5128 (2001).

${ }^{22}$ D. R. Hamann, Phys. Rev. 158, 570 (1967); P. E. Bloomfield and D. R. Hamann, Phys. Rev. 164, 856 (1967).

${ }^{23}$ K. Kobayashi, H. Aikawa, A. Sano, S. Katsumoto and Y. Iye, Phys. Rev. B 70, 035319 (2004).

${ }^{24}$ K. Kang, S. Y. Cho, J. J. Kim and S. C. Shin, Phys. Rev. B 63, 113304 (2001); M. E. Torio, K. Hallberg, A. H. Ceccatto and C. R. Proetto, Phys. Rev. B 65, 085302 (2002); A. A. Aligia and C. R. Proetto, Phys. Rev. B 65, 165305 (2002).

${ }^{25}$ I. Maruyama, N. Shibata and K. Ueda, cond-mat/0410061.

${ }^{26}$ A. Aharony, O. Entin-Wohlman and Y. Imry, Phys. Rev. Lett. 90, 156802 (2003) and references therein.

${ }^{27}$ N. E. Bickers, Rev. Mod. Phys. 69, 845 (1987), Table IX.

28 D. C. Langreth, Phys. Rev. 150, 712 (1966).

${ }^{29}$ F. D. M. Haldane, Phys. Rev. Lett. 40, 416 (1978).

${ }^{30}$ O. Entin-Wohlman and A. Aharony, unpublished.

${ }^{31}$ D. Ralph, Private communication.

${ }^{32}$ A. Aharony, O. Entin-Wohlman, B. I. Halperin and Y. Imry, Phys. Rev. B 66, 115311 (2002). 